\title{
Sobre El Abuso de Lo Necesario A Posteriori ${ }^{\mathrm{I}}$
}

\author{
Rafael Miranda Rojas ${ }^{2}$
}

\begin{abstract}
Resumen: El presente escrito argumenta cómo el denominado abuso de lo necesario a posteriori, propuesto en Beebee y Sabbarton-Leary (2010), asume erróneamente que: a) los enunciados necesarios a posteriori requieren un compromiso metafísico con un esencialismo no trivial; y b) que el experimento mental de la tierra gemela cumple un rol evidencial en la determinación de lo necesario a posteriori. Contra a), se sostiene que lo necesario a posteriori no requiere un compromiso esencialista no trivial; a lo sumo, el esencialismo no trivial es consecuencia de la propuesta de lo necesario a posteriori. Contra b), se afirma cómo el experimento mental de la tierra gemela cumple un rol subsidiario, explicativo y no evidencial. Esto evita un compromiso ontológico con el nexo concebible - posible. Luego, se discute cómo la noción de co- referencialidad rígida (de jure - de facto) es una vía de solución a la exigencia de esencialismo no trivial. Finalmente, se considera de qué modo el esencialismo científico de Brian Ellis, al enunciar condiciones a priori de satisfacción, no afecta la justificación de lo necesario a posteriori. Del mismo modo, se defiende un nexo término rígido de jure/clase sustancial - término rígido de facto/clase propiedad.
\end{abstract}

Palabras Clave: Necesidad. A posteriori. A priori. Esencialismo. Experimento Mental.

${ }^{1}$ La redacción de este escrito se enmarca en el proyecto post doctoral CONICYT FONDECYT N ${ }^{\circ}$ 3140174. Una versión de este escrito fue presentada en la III Latin American Analytic Philosophy Conference (ALFAn) \& Conference of the Brazilian Society for Analytic Philosophy (SB $\varphi$ A), $27-30$ de Mayo, 2014, Fortaleza (Brasil) y el IV Coloquio de Metafísica Analítica, 8 - 10 Octubre de 2014, Santiago (Chile). Sean mis agradecimientos para Javier Vidal López.

http://dx.doi.org/10.1590/S0101-31732017000100011

${ }^{2}$ Licenciado en Filosofía y Educación por la Pontificia Universidad Católica de Valparaíso, Chile (2007). Doctor en Filosofía por la misma Universidad (2012). Recientemente finalizó su proyecto postdoctoral FONDECYT (2014 - 2016) en la Universidad Católica del Maule, Chile. Académico de la Facultad de Ciencias Religiosas y Filosóficas de la Universidad Católica del Maule, Chile. Actualmente se desempeńa como director y docente del Magíster en Ciencias Religiosas y Filosóficas de la misma universidad (Línea de Investigación Filosofía del Lenguaje y Epistemología). Profesor Visitante del Magíster en Filosofía de la Universidad de Concepción, Chile. Miembro de la Asociación Chilena de Filosofía (ACHIF), la Asociación Latinoamericana de Filosofía Analítica (ALFAn), la Sociedad Chilena de Filosofía Analítica (SChFA) y la Sociedad Chilena de Filosofía Cristiana (SChFC). Sus áreas de estudio son: Filosofía Analítica, Filosofía del Lenguaje, Epistemología y Metafísica. Sus últimas publicaciones son los ensayos: "Enunciados de identidad, invariabilidad proposicional y estipulación contextual” (Revista Discusiones Filosóficas, v. 14, n. 23. Universidad de Caldas, Colombia. 2013b); "Consideraciones en torno al rechazo de la premisa in intellectu en el argumento ontológico" (Revista Temas Medievales, n. 21, 2013, Departamento de Investigaciones Medievales del Instituto Multidisciplinario de Historia y Ciencias Humanas [DIMED-IMHICIHUCONICET] Buenos Aires, Argentina); "Bootstrapping y justificación a priori" (por venir) Revista Discusiones Filosóficas. v. 16, n. 25, 2014. Universidad de Caldas, Colombia. 2014); y "Enunciados necesarios a posteriori, necesidad débil y racionalismo" (por venir) Revista Ideas y Valores, v. 65, n. 160, abril 2016. Universidad Nacional de Colombia. Bogotá, Colombia). E -mail: rafaelmirandarojas@ gmail.com; rmiranda@ucm.cl 


\section{INTRODUCCIÓN}

Recientemente, Helen Beebee y Nigel Sabbarton-Leary (2010, p. 159 - 179) han cuestionado lo que ellos denominan un abuso de lo necesario $a$ posteriori. Tal abuso consiste en que no se tienen argumentos para explicar porqué ciertos enunciados verdaderos, conocidos a posteriori, son también necesarios. Es lo que Albert Casullo (2003, p. 191) denomina estatus modal específico (specifical modal status). La idea intuitiva de esta noción es que la justificación del conocimiento de la verdad de cierto enunciado E es distinta de la justificación del conocimiento del estatus modal del mismo enunciado E. Así, el abuso consistiría en sostener que "Abuso de lo necesario a posteriori: La justificación del conocimiento de la verdad de ciertos enunciados de identidad teórica fundamenta la justificación del conocimiento de que tales enunciados son necesarios".

Según los autores, este paso exigiría un compromiso con un tipo de esencialismo no trivial, el denominado esencialismo científico (Scientific Essentialism) (ELLIS, 2001) ${ }^{3}$. Siendo el esencialismo científico un caso de esencialismo no trivial, se propone en este escrito que lo necesario a posteriori no depende de alguna tesis esencialista de este tipo, sino sólo de un esencialismo trivial que permita afirmar la necesidad del principio de identidad y de diferencia (MIRANDA, por venir).

El desarrollo de este escrito es el siguiente: La sección 2 analiza la tesis implícita que permite afirmar el denominado abuso de lo necesario a posteriori. Ulteriormente, se enuncia y fundamenta la tesis guía de este escrito, a saber: Lo necesario a posteriori no depende de una tesis esencialista no trivial. La ventaja de esta tesis es que no exige un compromiso esencialista específico. Del mismo modo, se analiza la denominada primera ruta kripkeana y la importancia de que la co - referencialidad rígida no sea comprendida como mera cuestión de hecho. Para evitar esto, se enuncian las condiciones que debe cumplir la co - referencialidad rígida. La sección 3 especifica las razones de porqué fracasa la denominada segunda ruta kripkeana propuesta por Beebee y Nigel Sabbarton-Leary (2010), siendo la más importante la comprensión del rol que le corresponde al experimento mental. Se critica el rol evidencial, y se propone el rol heurístico. Siguiendo a Roca - Royes (2011), se enfatiza la importancia del criterio de carencia de conocimiento (lack of knowledge) y el

\footnotetext{
${ }^{3}$ La tesis central de éste esencialismo es que las leyes naturales son metafísicamente necesarias, pero cognoscibles sólo a posteriori. Este escrito no discute esta tesis metafísica.
} 
error que constituye el comprenderlo como un caso de simulación (pretense). La principal consecuencia es que se evita un compromiso ontológico con el nexo concebible - posible. Posteriormente, se discute el experimento mental de la tierra seca, y cómo su crítica modal al externalismo exige comprender el experimento mental como evidencia. La sección 4 considera el estatuto epistémico a posteriori de los enunciados de identidad teórica, y cómo el esencialismo científico propuesto por Ellis no cumple este requisito de a posterioridad. Se rescata, no obstante el nexo clase sustancial/clase propiedad como un caso análogo del nexo rigidez de jure/rigidez de facto, pero evitando el compromiso con un esencialismo no trivial. Finalmente, se enuncian las principales conclusiones de este escrito.

\section{FUNDAMENTACIÓN DEL ABUSO, DISTINCIÓN NECESARIO A POSTERIORI/ESENCIAL Y CO -REFERENCIALIDAD RÍGIDA DE JURE - DE FACTO}

Beebee y Nigel Sabbarton-Leary han explicitado lo que ellos denominan un abuso de lo necesario a posteriori al inicio de su escrito: "[...] contemporary metaphysicians have increasingly appealed to the category of the necessary a posteriori. What they generally fail to do, however, is provide any argument for why the truths in question fall into this category." (BEEBEE; SABBARTON-LEARY, 2010, p. 159).

El problema no está, por tanto, en la verdad de los enunciados de identidad teórica, sino más bien en la justificación que permita sostener que tales enunciados son necesariamente verdaderos ${ }^{4}$. La necesidad que se está

\footnotetext{
${ }^{4}$ Ver Kant (1965, p. 43-44): "Experience teaches us that a thing is so and so, but not that it cannot be otherwise. First, then, if we have a proposition which in being thought is thought as necessary, it is an a priori judgement [...] Necessity and strict universality are thus sure criteria of a priori knowledge." Kripke (1980, p. 159) observa cómo este modo de entender el nexo a priori - necesario, impide proponer enunciados necesarios a posteriori. Destaca Kripke la importancia de la distinción puede debe (esto es discutido en MIRANDA, por venir): "Kant thus appears to hold that if a proposition is known to be necessary, the mode of knowledge not only can be a priori but must be." (ibid. Énfasis de quién escribe) Las consecuencias de este análisis, para la viabilidad de enunciados a posteriori es central. No se trata de que Kripke confunda el acceso a posteriori con la justificación a posteriori: se trata de que los enunciados necesarios a posteriori son un caso análogo al de los enunciados matemáticos, de modo tal que si un sujeto $\mathrm{S}$ conoce a posteriori que un enunciado $\mathrm{E}$ es verdadero, por tratarse de un tipo especial de enunciado, ese sujeto $S$ conoce a posteriori que $E$ es necesariamente verdadero. Así lo sostiene líneas después Kripke: "[...] one can learn a mathematical truth a posteriori by consulting a computing machine, or even by asking a mathematician. Nor can Kant argue that experience can tell us that a mathematical proposition is true, but not that it is necessary ; for the peculiar character of mathematical propositions (like Goldbach's conjecture) is that one knows (a priori) that they cannot be contingently true; a mathematical statement, if true, is necessary. All
} 
cuestionamiento aquí es la necesidad de re, necesidad metafísica sobre los objetos que son designados. Beebee y Sabbarton - Leary observan que los términos que se encuentran en la derecha de los enunciados de identidad teórica pueden ser comprendidos como descripciones de la naturaleza subyacente (underlying nature) de la clase natural, pero al costo de que la determinación de esta naturaleza subyacente como la esencia de dicha clase natural dependa de un experimento mental similar al de la tierra gemela (PUTNAM (1975). Sin este experimento mental, se carecería de la fundamentación para comprender la naturaleza subyacente como esencia de la clase natural: "[...] what is discovered a posteriori is merely the underlying nature of the kind in question, rather than its essence; and this possibility will in turn need to be removed using a Twin Earth-style thought experiment." (BEEBEE; SABBARTONLEARY, 2010, p. 161).

the cases of the necessary a posteriori advocated in the text have the special character attributed to mathematical statements: Philosophical analysis tells us that they cannot be contingently true, so any empirical knowledge of their truth is automatically empirical knowledge that they are necessary. This characterization applies, in particular, to the cases of identity statements and of essence. It may give a clue to a general characterization of a posteriori knowledge of necessary truths." Cabe notar que, distinto al modo cómo lo entienden Gendler \& Hawthorne (2002, p. 30), estos enunciados de identidad si requieren de información empírica que establezca que son enunciados de identidad verdaderos y, por esto, necesarios. Los autores sostienen explícitamente que: "Whether a proposition is necessary or contingent is a metaphysical question: it turns on whether its truth is independent of what the world is like." (GENDLER; HAWTHORNE, 2002, p. 30). Estrictamente, los enunciados de identidad considerados en la defensa de lo necesario a posteriori, no son independientes del modo cómo es el mundo. Esta lectura, en rigor anti kripkeana, continúa líneas después, al sostener de los casos "Héspero es Fósforo" y "Agua es H2O" que: "They are necessary because the rigid designators on each side of the copula co - refer in the actual world, in hence in all possible worlds; but they are knowable only a posteriori because the co - reference of the terms is itself contingent" (GENDLER; HAWTHORNE, 2002, p. 31) La co - referencia rígida no es del caso de que sólo ocurre (happen) qué es co - referencial. Es un error que esto sea comprendido de ese modo, si se considera lo explícitamente sostenido por Kripke (1971, p.136):

“(2) (x) $\square(\mathrm{x}=\mathrm{x})$

But

(3) $(\mathrm{x})(\mathrm{y})(\mathrm{x}=\mathrm{y}) \rightarrow[\square(\mathrm{x}=\mathrm{x}) \rightarrow \square(\mathrm{x}=\mathrm{y})]$

...From (2) and (3), we can conclude that, for every $x$ and $y$, if $x$ equals $y$, then, it is necessary that $x$ equals y:

(4) (x) (y) (x = y) $\rightarrow \square(\mathrm{x}=\mathrm{y})$ ”

Lo anterior se enmarca en la discusión sobre la ley de sustitución de la identidad (law of the substitutivity of identity). Es correcto sostener que la postura kripkeana no acepta como tesis subsidiaria que la co - referencia de los términos rígidos sea contingente, pese a que lo enunciado en la primera parte del párrafo citado de Gendler \& Hawthorne (2002) pueda ser entendido como una postura actualista que soluciona lo afirmado líneas después (MIRANDA, 2011). 
La implausibilidad de sostener que el experimento mental de la tierra gemela sea la fundamentación de lo necesario a posteriori, permite observar el avance que supone distinguir dos nociones centrales en la postura kripkeana, a saber: esencialismo/necesario a posteriori. Una guía de esta distinción es enunciada por Kripke": "It's certainly a philosophical thesis, and not a matter of obvious definitional equivalence, either that everything a priori is necessary or that everything necessary is a priori.” (KRIPKE, 1980, p. 35).

Este escrito propone, análogamente, que “i) Es una tesis filosófica (y no un asunto de equivalencia de definición) que lo necesario a posteriori exige una tesis esencialista no trivial".

Esta tesis es desde ya plausible al considerar el caso de los enunciados de identidad en términos singulares, al no exigir tesis esencialista subsidiaria para afirmar la necesidad de casos como "Héspero es Fósforo". Sin embargo, los enunciados de identidad teórica exigen una distinción de jure/de facto, debido a que aceptar una doble estipulación exige que ambos términos rígidos sean de jure y, de este modo, cognoscibles a priori. La co - referencialidad rígida de jure - de facto es una vía de solución a este problema. Si esto es correcto, se puede afirmar que lo necesario a posteriori se establece vía co - referencialidad rígida de jure - de facto, independiente de algún compromiso esencialista ulterior. Un antecedente del nexo esencialista - necesario a posteriori se observa en el siguiente análisis que Casullo (2003) realiza de la postura enunciada en Kripke (1980 y 1971):

(Tl) The concepts of a priori truth and necessary truth are different; they are not interchangeable.

(T2) It is a substantive philosophical thesis, one that requires philosophical argument to establish, that everything necessary is a priori or that everything a priori is necessary.

(T3) The a priori and the necessary are not coextensive; there are examples of necessary a posteriori truths and probably contingent a priori truths. (CASULLO, 2003, p. 181).

Es ampliamente aceptado que, independiente de la discusión de lo necesario a posteriori en los enunciados de identidad teórica, es plausible sostener que (T3) ya presenta casos en los enunciados de identidad que incluyen términos singulares co - referenciales (MIRANDA, 2013), por lo

${ }^{5}$ Luego desarrollada por Casullo (2003). 
que el que la discusión se centre en los primeros se debe, en gran parte, a que no es evidente qué objeto es el referente de los términos generales. Si casos como "Héspero es Fósforo" son reconocidos como necesarios a posteriori ${ }^{6}$, la pregunta que persiste es porque casos como "El oro es la sustancia cuyo número atómico es 79" o "Agua es $\mathrm{H} 2 \mathrm{O}$ ” no son admitidos del mismo modo. Casullo, tal como luego proponen Helen Beebee y Nigel Sabbarton-Leary (2010) atribuye esto a una tesis esencialista no trivial. No obstante, esto no coincide estrictamente con la postura kripkeana. Kripke afirma que una postura esencialista depende de la separación, de la división entre a priori a posteriori y necesario - contingente. Esta distinción es, en otras palabras, condición necesaria para sostener un esencialismo no trivial. Lo necesario a posteriori viene a ser un criterio a favor del esencialismo, no el esencialismo un criterio que fundamente lo necesario a posteriori.

If the essentialist view is correct, it can only be correct if we sharply distinguish between the notions of a posteriori and a priori truth on the one hand, and contingent and necessary truth on the other hand, for although the statement that this table, if it exists at all, was not made of ice, is necessary, it certainly is not something that we know a priori. (KRIPKE, 1971, p. 152-153).

En otras palabras, una postura esencialista no debería ser comprendida como la fundamentación de enunciados necesarios a posteriori. Esto es controversial por diversas razones, siendo la central el que se tenga por un objetivo subsidiario de la propuesta de lo necesario a posteriori la defensa de cierto esencialismo. Sea afirmativa o negativa la postura ante este problema, para refutar el aludido abuso se debe afirmar que:

a) Lo necesario a posteriori no depende de una tesis esencialista no trivial.

${ }^{6}$ Ver Haukioja (2012), quién defiende una independencia de lo necesario a posteriori y la noción de rigidez, y sostiene que la base para los enunciados de identidad teórica descansa en una dependencia de actualidad (actuality-dependence). Si bien la propuesta de Haukioja intenta diferenciar rigidez dependencia de actualidad, es claro en el inicio de su escrito que, al menos, rigidez supone dependencia de actualidad: "[...] given that "Hesperus" and "Phosphorus" are rigid designators, and given that they are coreferential in the actual world, it follows that they designate the same object in all possible worlds." (HAUKIOJA, 2012, p. 400) Del mismo modo, Glüer \& Pagin (2012) defienden que la noción de rigidez no puede explicar el estatuto epistémico a posteriori de los denominados enunciados de identidad teórica, sino sólo su estatuto modal, su necesidad. Para explicar este estatuto, proponen lo que ellos denominan switcher-style dual-property association, asociación que enfatiza el rol de la naturaleza subyacente (underlying nature) en la determinación de lo necesario a posteriori (GLÜER; PAGIN, 2012, p. 160). 
Esto no es, strictu sensu, antiesencialismo. Por ejemplo, es errado afirmar que Kripke no defiende un esencialismo de origen, o que no defiende un dualismo anti fisicalista. Sólo es trivial la distinción necesario - esencial, si se alude a un esencialismo trivial, como es el caso de la identidad consigo mismo (self identity). Por lo tanto, la tesis propuesta tiene por objetivo distinguir lo necesario a posteriori de un esencialismo no trivial, sin que ello exija rechazar un nexo ulterior con este tipo de esencialismo ${ }^{7}$. Proponer esta distinción tiene una ventaja epistémica y metafísica:

b) No exige al defensor de lo necesario a posteriori un compromiso específico con algún tipo de esencialismo.

c) Impide que la postura anti esencialista ataque la noción de necesario a posteriori, al derrotar (defeat) cualquier tipo de esencialismo no trivial.

Algunos autores han propuesto que este debate necesario a posteriori/ esencial podría evitarse si, de modo similar al caso de los enunciados de identidad para singulares, los términos que determinan como necesario un enunciado de identidad teórica ${ }^{8}$ fuesen ambos nombres propios, como es en

7 Por ejemplo, Della Rocca (2002, p. 226, n. 4) no distingue entre necesario - esencial. No obstante, ulteriormente Della Rocca sostendrá que el esencialismo que critica acepta la siguiente tesis:

"(b) The modal properties of an individual (properties such as being essentially F or possibly G) are had independently of the way in which the individual is referred to." (DELLA ROCCA, 2002, p. 226). Y no (a), a saber:

“(a) Individuals have some properties essentially or necessarily” (DELLA ROCCA, 2002, p. 226.)

La razón principal por la que la autora centra su crítica en (b) y no en (a), es debido a que le parece que el rechazo de (a) exige un anti esencialismo absoluto, tal que ninguna propiedad de un objeto o es necesaria a tal objeto o. Este anti esencialismo absoluto descarta, por lo tanto, un esencialismo trivial. Respecto a (b), la razón principal por la que la autora la rechaza es debido a que le parece, en última instancia, errado sostener que las propiedades modales son independientes del modo cómo es referido el objeto o. Si bien Della Rocca atribuye esta tesis a Kripke, cabe destacar que la noción de rigidez es precisamente un modo en que el objeto es referido, y la necesidad de los enunciados de identidad teórica, y de los enunciados necesarios a posteriori, descansa en la rigidez de los términos que refiere dicho objeto (o clase). Más importante para el objetivo de este escrito, la autora relaciona esta tesis (b) con la necesidad de la identidad: "Let us say that 'a' is a name for a particular object [...] a has the property of being necessarily self - identical. To deny this would be to hold that a could fail to be identical with itself, an absurd claim." (DELLA ROCCA, 2002, p. 227) Es correcto, como se observa en la nota 1 de este escrito, que la sustitución de los idénticos le permite a Kripke afirmar no sólo un esencialismo trivial (que descanse en esta propiedad de auto identidad) sino también la exigencia de que toda propiedad que le corresponda a x, le corresponda a y (nótese que esto no ha de ser entendido como un caso de hiper- esencialismo, pues no exige que toda propiedad de $\mathrm{x}$ ni de $\mathrm{y}$, que son el mismo objeto, sea necesaria).

${ }^{8}$ En tanto casos de enunciados de identidad, es una tesis subsidiaria de este escrito (defendida en MIRANDA, por venir) que los enunciados de identidad teórica son necesariamente verdaderos (si son verdaderos) en base al principio de identidad y, por lo tanto, de un principio a priori. Esto es 
efecto en "Pablo Neruda es Neftalí Reyes", sin que ello derive en sostener que, si bien necesarios, enunciados de este tipo no serían a posteriori. Beebee y Nigel Sabbarton-Leary (2010, p. 160) observan no obstante una distinción entre ambos términos, en el caso de los enunciados de identidad teórica. Los de la izquierda son:

(i) non-descriptive, (ii) de jure rigid e (iii) introduced by ostensive baptism.

Los de la derecha, en contraste, son:

(i) definite descriptions, (ii) de facto rigid y (iii) discovered and described by science rather than ostensively introduced.

Todo sugiere que el peso de la fundamentación a posteriori recae en aquellos términos que no son meramente estipulados por un bautismo ostensivo, los de la derecha. La razón principal para sostener esto es que, a diferencia de los casos de identidad en singulares, pareciese que sostener la tesis de que ambos términos son estipulados ostensivamente, deriva en un compromiso a priorista que, al fundamentar un enunciado $\mathrm{E}$ como necesario a posteriori, presenta el problema de cómo dos estipulaciones no han de ser comprendidas como arbitrarias y a priori. Esta sería, en el análisis de los autores, la denominada primera ruta de lo necesario a posteriori. Esta es la ruta que precisamente descartan Beebee y Nigel Sabbarton-Leary (2010, p. 160) al sostener que:

One route is analogous to the route Kripke describes in the case of proper names. If one can plausibly maintain that the two terms in the 'identity' statement are both analogous to proper names (as perhaps 'gold' is, but 'the element with atomic number 79' clearly—we believe-is not), whose reference traces back to two distinct baptismal events which happen to name the same kind, then one has a good case for claiming that the relevant identity claim is necessary a posteriori.

El análisis de esta cita permite enunciar, contra Beebee y SabbartonLeary, que:

d) el no comprender los términos de la derecha como nombres propios no exige que toda descripción definida sea comprendida como rígida (un

particularmente importante, considerando lo afirmado en la tesis a), pues permite sostener cómo estos enunciados no requieren de un compromiso esencialista no trivial, al sostener la necesidad a posteriori. 
descriptor ${ }^{9}$, como lo destacan ulteriormente los autores, siguiendo a Connelly et al. (2005)).

e) la co - referencialidad rígida no puede ser comprendida como algo que solo ocurre (happen)

Pues ello conduce a la crítica respecto a si dos términos rígidos que pueden no designar el mismo objeto en toda situación contrafáctica (renatecordate) son un ejemplo de un enunciado necesario a posteriori. Si dos términos rígidos designan el mismo objeto o la misma clase, no debe ser sólo una cuestión de hecho. Es una tesis intuitiva que, si la co - referencialidad fuese sólo una cuestión de hecho, no sería rígida. Por otra parte, el rechazo de co referencialidad rígida permite al menos de entrada sostener que los enunciados de identidad no son necesariamente verdaderos. Se pueden enunciar tres condiciones que han de cumplir términos co - referenciales rígidos:

C1) Dos términos $\mathrm{T} 1$ y $\mathrm{T} 2$, independiente uno de otro ${ }^{10}$, designan rígidamente un objeto $\mathrm{O}$ o clase $\mathrm{C}$.

\footnotetext{
${ }^{9}$ Una característica central de un descriptor es no cumplir con el denominado criterio de la naturalidad (naturalness). El ejemplo dado por los autores (Idem, 1) es lo que estos denominan NARG u "Object bigger than a car". Es una descripción que cumple cualquier objeto más grande que un auto, sin criterio natural unificador. La Porte (2000, p. 299) denomina a estas clases, non-natural. Considérese el ejemplo "Mary favourite color" ¿Es rígida esta descripción? ¿Selecciona el mismo color en toda situación contrafáctica? Esto ha sido discutido, en particular, por aquellas posturas que defienden una lectura de los términos de clases naturales como predicados (MARTÍ, 2004, p. 133). Si bien no es la lectura que defiende este escrito, conviene tener presente la distinción propiedad determinada - propiedad determinable. En el caso del color favorito de Mary, puede plantearse plausiblemente que esta descripción corresponde a una propiedad a) determinable (color), no b) determinada (azul, rojo, verde, etc...). De este modo, la rigidez sería correctamente sostenida en a), pero no en b). Esto conduce a la implausible tesis siguiente: 1) Toda propiedad determinable es rígida y 2) Toda propiedad determinada no es rígida. Es implausible, pues la propiedad determinable ha de tener una propiedad determinada específica. Por otra parte, tal como destaca Haukioja (2012, p. 401), la discusión sobre la rigidez en los términos que designan colores deriva en el debate esencialismo - antiesencialismo: "There may be room for discussion about whether colour terms in fact are rigid, but if the essentialist view were correct, there should be no doubt whatsoever about their status: in general, red things are obviously not essentially red." Tal vez, considerando el aporte de La Porte, podría evitarse esta consecuencia implausible, apelando a la no naturalidad. Sin embargo, esto sólo es viable en casos como el del color favorito de Mary, en que el color no es designado directamente. En última instancia, eso es otra razón para diferenciar (si bien no separar, al menos en Kripke) lo necesario a posteriori de una tesis esencialista.
}

${ }^{10}$ Cf. Miranda (2013). Es central comprender que la rigidez de ambos términos T1 y T2 no es dependiente de su co - referencialidad rígida. Esto permite sostener la tesis de invariabilidad proposicional en casos paradigmáticamente complejos, como "Neftalí Reyes es Neftalí Reyes" y "Neftalí Reyes es Pablo Neruda". 
C2) La rigidez de T1 y T2 exige que, en caso de designar el mismo objeto $\mathrm{O}$ o clase $\mathrm{C}$, tal co - referencialidad sea rígida.

C3) La co - referencialidad rígida descarta co - referencialidad contingente (descarte de la alternativa just happen o mera cuestión de hecho).

\title{
3 LA SEGUNDA RUTA
}

Beebee y Nigel Sabbarton-Leary plantean que el problema de la falta de argumentos de porqué los enunciados de identidad teórica son necesarios, si son verdaderos, se da en lo que ellos denominan la segunda ruta de lo necesario a posteriori, la que debiese justificar dos aspectos: la necesidad y la a posterioridad:

\begin{abstract}
The other route is the route we attribute to Kripke above in the case of natural kind terms... Here, the term on the left ("gold") is analogous to a proper name and names a natural kind, and the term on the right ("the element with atomic number 79") specifies the essence of that kind. (BEEBEE; SABBARTON-LEARY, 2010, p. 161, grifo do autor).
\end{abstract}

Esto, que parece obvio, es el centro de la crítica de los autores, pues es el supuesto de que ambos criterios se cumplen, lo que conduce a sostener que los enunciados de identidad teórica son necesarios, y son a posteriori. Lo que exigen los autores es un caso: "But one must argue that one's alleged case of a necessary a posteriori truth fits this model." (BEEBEE; SABBARTONLEARY, 2010, p. 161). El caso que atacan es el de "agua". El centro de la crítica pretenden los autores que sea la incidencia del experimento mental como argumento, lo que será analizado en la sección siguiente. Pese a esto, se puede destacar un contraste entre lo que los autores entienden como la ruta kripkeana, y lo que los autores sostienen puede atribuirse a esta ruta kripkeana:

Let's grant that water has a straightforward underlying nature: actual samples of water are samples of a substance that is composed of molecules, each of which is composed in turn of two hydrogen atoms and one oxygen atom. It does not follow, however, that "water is $\mathrm{H} 2 \mathrm{O}$ " is necessary, because it does not follow that the underlying nature is water's essence. For of course it might be, for all that has been said so far, that the term "water" is not analogous to a proper name: it might have a meaning such as "whatever potable liquid is typically to be found in rivers and lakes and falls from the sky", in which case the claim that water is $\mathrm{H} 2 \mathrm{O}$ would be contingent rather than necessary. In order to rule this possibility out, we have to run a 
Twin Earth-style thought experiment: it is only the fact that we (allegedly) intuitively judge that XYZ is not water, despite meeting the above description, that justifies the claim that water's underlying nature is its essence, and hence that "water is H2O" is necessary. (BEEBEE; SABBARTON-LEARY, 2010, p. 161, grofo do autor).

Es importante desglosar esta cita, y contrastarla con lo explícitamente aceptado por los autores como la segunda ruta kripkeana (BEEBEE; SABBARTON-LEARY, 2010, p. 161). Primero, según los autores: ¿Cuál es la razón por la que no se sigue que "Agua es $\mathrm{H} 2 \mathrm{O}$ " es necesaria? La razón es que la naturaleza subyacente (underlying nature) no es la esencia de agua (water's essence) Segundo: ¿Cuál es el fundamento para establecer esta distinción entre underlying nature y water's essence, según los autores? El fundamento es que el término "agua" puede no ser un análogo de un nombre propio (For of course it might be, for all that has been said so far, that the term 'water' is not analogous to a proper name), y tener por contraparte un significado como "cualquier líquido potable que es típicamente encontrado en ríos y lagos y cae desde el cielo" ('whatever potable liquid is typically to be found in rivers and lakes and falls from the sky'). Debiese ser evidente como este paso es inviable, ya que se acepta explícitamente la comprensión del término "agua" como análogo a un nombre propio en la denominada segunda ruta kripkeana, lo que descarta la posibilidad de que "agua" sea una descripción definida disfrazada. Esta fundamentación, sin siquiera considerar aún el problema del experimento mental, no respeta una condición fundamental de los enunciados de identidad kripkeanos. Apoyados en esta posibilidad epistémica descartada extensamente por Kripke (1980), los autores dan paso al cuestionamiento del experimento mental.

\subsection{El PROBLema DEL EXPERIMENTO MENTAL}

Un experimento mental se comprende como un supuesto estado de cosas (por lo general una situación contrafáctica) ${ }^{11}$ a partir del cuál se intenta evidenciar la plausibilidad de cierta postura, ante las consecuencias

\footnotetext{
${ }^{11}$ Brown (2011) distingue entre experimento mental y razonamiento contrafáctico, pues observa que el segundo requiere por regla general experimentación: "They should also be distinguished (N.A: los experimentos mentales) from counterfactual reasoning in general, as they seem to require an experimental element)." Para que la distinción sea importante, se asume que esta experimentación es de carácter fáctica.
} 
contraintuitivas que tendría su rechazo ${ }^{12}$. En el caso de los enunciados necesarios a posteriori, Beebee y Nigel Sabbarton-Leary (2010, p. 161) cuestionan que sea un experimento mental el argumento que fundamente que ciertos hechos no sólo ocurren, sino que ocurren necesariamente:

Kripke invites us to imagine a scenario where there is a substance that has a "completely different atomic structure from that of water, but resembled water in these [characteristic] respects" (Kripke 1980, 128),); is this water? According to Kripke the answer is no. Just "as there is a fool's gold there could be a fool's water" (ibid.). Given that water is $\mathrm{H} 2 \mathrm{O}$, nothing lacking that atomic structure could be water.

La crítica al experimento mental hecha por los autores constituye un cuestionamiento implícito a que las intuiciones que tenga cierto sujeto $S$ sean una base correcta para establecer la necesidad de ciertos enunciados. Hay, por lo menos, cierta ambigüedad en la postura kripkeana ante el rol de las intuiciones. Por un lado, Kripke (1980, p. 46) las comprende positivamente, como una guía epistémica válida para establecer cierto conocimiento. Por otro, acepta que en ciertas ocasiones conducen a lo que Gendler \& Hawthorne (2002, p. 33) denominan la ilusión de posibilidad:

[...] he (Kripke) does offer a strategy for re-establishing a link between intuitions of possibility and what is in fact posible. There will, he acknowledges, be cases where it seems... to be possible that not-P, but where, in fact, $\mathrm{P}$ is necessary: a posteriori necessities provide us with a class of such cases. In such cases, we will be faced with an illusion of possibility - not-P will seem possible, though in fact it is not. ${ }^{13}$

En particular, el experimento mental sostiene la plausibilidad de que, si es el caso que "Agua es $\mathrm{H} 2 \mathrm{O}$ " es verdadero, sería contradictorio sostener como verdadero "Agua no es H2O"14, y es implausible sostener que "Agua es XYZ"

\footnotetext{
${ }^{12}$ Siguiendo la taxonomía propuesta por Popper (1959), este tipo de experimento mental corresponde a un caso de experimento apologético, que tiene por finalidad dar razones a favor de una teoría. Los otros dos son heurístico, cuyo objetivo es ilustrar una teoría, y crítico que tiene por objetivo dar razones contra una teoría. Este escrito sostiene que el caso de la tierra gemela cumple un rol heurístico.

${ }^{13}$ Ver Bealer (2002, p. 82). El conflicto entre intuiciones, sostiene el autor, se debe a que al menos una de ellas está mal informada (misreported). Esto, como se discutirá, es un caso de lack of knowledge.

${ }^{14}$ Ver Roca-Royes (2011). La autora atribuye una comprensión de lo concebible, que explicita una consecuencia de este tipo, a Yablo (1993, p. 29). Observa la autora en Yablo un caso de lo que ella denomina (siguiendo a Worley 2003, p. 17). epistemic conceivability-based account (lo concebible fundado epistémicamente), en contraste con casos del tipo no epistémico (non epistemic conceivability-
} 
no exige que "Agua no es $\mathrm{H} 2 \mathrm{O}$ ” es verdadero.

Es importante fijarse en un aspecto de esta aludida contradicción: Permite sostener la necesidad del enunciado "Agua es H2O", si es que resulta ser el caso (a posteriori) que:

i) Agua es efectivamente $\mathrm{H} 2 \mathrm{O} y$

ii) $\mathrm{H} 2 \mathrm{O}$ es una propiedad que se aplica a un y sólo un objeto, o clase.

Efectivamente, 2) es la característica central que distingue una descripción definida rígida de facto, de una descripción definida simpliciter (y de una descripción rigidificada con el operador de actualidad). El cuestionar el experimento mental es sólo un caso de una crítica más profunda: el si la noción de lo concebible (conceivable) es una vía adecuada para el conocimiento de posibilidades de re, si es una vía para establecer como válida la modalidad de re. Kripke asume el acceso epistémico a verdades necesarias de re, y para ello acepta que se puede predicar, de objetos y clases, propiedades modales. La intuición básica es que hay hechos contingentes, como que Aristóteles fue el discípulo de Platón, y hechos necesarios, como que Aristóteles es idéntico a sí mismo. Si un enunciado de identidad teórica expresa un hecho como el segundo ( $y$ al ser un enunciado de identidad no parece ser de otro modo, ver nota 2), se puede aseverar que la necesidad a posteriori está correctamente fundamentada, sólo en esencialismo trivial. Es una consecuencia ulterior el que esta identidad consigo mismo (selfidentity) permita sostener la distinción entre accidental - esencial.

Recientemente, Sonia Roca-Royes (2011, p. 22-49) ha puesto en duda el rol de lo concebible en la determinación de lo posible. ${ }^{15}$ Anteriormente, Putnam realiza una crítica similar, al sostener que:

[...] we can perfectly well imagine having experiences that would convince us (an that would make it rational to believe that) water isn't $\mathrm{H} 2 \mathrm{O}$. In

based account). La diferencia central es que, en el caso fundado epistémicamente, lo que un sujeto $\mathrm{S}$ conoce o cree determina lo que es concebible. El caso del tipo no epistémico, comprende como concebible lo que es verdadero en al menos un mundo posible. De lo concebible alá Yablo afirma la autora: "The Greeks, according to Yablo, could conceiveY (N.A: Y indica que es lo concebible tal y como lo comprende Yablo) of water being other than $\mathrm{H} 2 \mathrm{O}$ (due to their lack of chemical concepts and their lack of knowledge that water is $\mathrm{H} 2 \mathrm{O}$ ). However, water is not $\mathrm{H} 2 \mathrm{O}$ is not conceivableY for a contemporary subject who knows that water is $\mathrm{H} 2 \mathrm{O}$ (the idea is that that would require conceiving of a contradiction: $\mathrm{H} 2 \mathrm{O}$ not being H2O)." (ROCA-ROYES, 2011, p. 24).

${ }^{15} \mathrm{El}$ objetivo central de la autora es cuestionar que lo concebible sea una vía para establecer qué es posible. Esto sigue la línea enunciada por Gendler \& Hawthorne (2002, p. 33): "[...] the necessary a posteriori seems to guarantee that there will be cases of conceivability without possibility." 
that sense, it is conceivable that water isn't $\mathrm{H} 2 \mathrm{O}$. It is conceivable but isn't logically possible! Conceivability is no proof of logical possibility...human intuition has not privileged access to metaphysical necessity. (PUTNAM, 1975b, p. 233).

Esto es importante, pues permite observar cómo un experimento mental puede permitir descartar que casos como "Agua es XYZ", por el sólo hecho de ser concebibles, constituyen situaciones contrafácticas posibles. El ejemplo presentado por la autora es el siguiente:

(1) Zombies_ physical duplicates of human beings that lack any phenomenal experience_-are conceivable.

(2) If $\mathrm{p}$ is conceivable, then $\mathrm{p}$ is possible. Therefore,

(3) Zombies are possible. (ROCA-ROYES, 2011, p. 25).

Considerando explicítamente el caso de Agua, afirma la autora: “[...] someone who does not know that water is $\mathrm{H} 2 \mathrm{O}$ can conceive $\mathrm{Y}$ of water being other than $\mathrm{H} 2 \mathrm{O}$, despite the fact that this is (metaphysically) impossible." (ROCA-ROYES, 2011, p. 25).

Analogar el caso de Zombie con $\mathrm{H} 2 \mathrm{O}$ muestra cómo lo concebible no debiese ser comprendido como un criterio que determine lo posible, al menos no en todos los casos (BEALER, 2002, p. 76) ${ }^{16}$. Si así fuese, en el supuesto de que un sujeto $S$ no tiene cierta información empírica, sería correcto dar el paso de:

\footnotetext{
${ }^{16}$ Sostiene el autor que una equivalencia absoluta entre "concebibilidad" y posibilidad es implausible, pues exige comprometerse con la siguiente tesis: "it is posible that p iff it is conceivable that p" Es implausible, pues las intuiciones que fundamentan lo que un sujeto $S$ entiende por concebible, son falibles. Más importante para este escrito, casos como el de la tierra gemela exigen que, aún si p es concebible, p sea estrictamente imposible. Ulteriormente, Bealer (2002, p. 80) destaca la distinción entre posibilidad epistémica y metafísica, tal que aún si el caso de la tierra gemela se comprende como posible, esta posibilidad es de carácter epistémico, no metafísico. Es lo que el autor denomina could-of-qualitative-evidential-neutrality. Ulteriormente, Bealer (2002, p. 107) defiende que el vacío modal (modal gap) que se presenta en los casos de enunciados de identidad teórica es solucionado vía intuiciones que no presenten el problema de estar mal informadas (misreported). El caso de "agua" es paradigmático, y si bien el autor observa una dependencia evidencial en el experimento mental, es correcto sostener que ese rol es dependiente de la información empírica que permite afirmar como mal informados casos como el de la tierra gemela. Finalmente, Bealer defiende un tipo de confiabilismo modal (modal reliabilism), que depende en gran medida de una estabilidad semántica fundada en la extensión de los términos de clase: "[...] if a simple of a given purely compositional stuff has such and- such composition, then, necessarily, all other samples of that purely compositional stuff also have that composition." (BEALER, 2002, p. 107.).
} 
iii) Sostener que es concebible que Agua es $\mathrm{XYZ}$ a

iv) Sostener que es posible que Agua es XYZ.

Pero este paso es cuestionable. A esta carencia de información empírica Sonia Roca - Royes (2011, p. 27) lo denomina lack of knowledge. Afirma la autora: "The Greeks could find it conceivable that Hesperus and Phosphorus occupy different positions in the sky because of their lack of knowledge that Hesperus is Phosphorus."

Un punto importante es que, acorde con la carencia de conocimiento, un sujeto $\mathrm{S}$ no debiese conocer que es verdadero el enunciado "Héspero es Fósforo". De allí la relevancia de la carencia de conocimiento. De modo análogo, sólo sería concebible que "Agua no es $\mathrm{H} 2 \mathrm{O}$ ", si no se conoce que es verdadero "Agua es H2O”. Kripke (1980, p. 103) defiende una postura cercana al sostener:

[...] there's one sense in which things might turn out either way, in which it's clear that that doesn't imply that the way it finally turns out isn't necessary... Obviously, the "might" here is purely "epistemic" -it merely expresses our present state of ignorance, or uncertainty.

Lo que intenta el experimento mental de la tierra gemela es precisamente establecer que, aunque concebible, es falso que Agua es XYZ. Se puede objetar, no obstante, que contradicciones de este tipo se presentan en otros casos, en los que el rechazo de la posibilidad parecería contraintuitivo. Considérese el siguiente condicional:

$$
\begin{aligned}
& \text { “(a) q; } \\
& \text { (b) if q then } \square \neg \text { p." (ROCA-ROYES, 2011, p. 27). }
\end{aligned}
$$

Si se entiende q por "Agua es H2O" y p por "Agua es XYZ”, lo que sostiene el condicional es que la verdad de q establece como necesariamente falso el enunciado p. La autora critica este condicional, basada en la noción de pretense $^{17}$, con el objetivo de cuestionar las implicancias esencialistas que

\footnotetext{
${ }^{17}$ Ver Roca-Royes $(2011$, p. 28): "[...] we first pretend that Oa,b,c and see whether, under the scope of that pretense, we find it conceivable that Oa,d,e." La esencialidad que está cuestionando aquí la autora es la esencialidad de origen (EO). Oa es el objeto, y en el primer caso es originado por b y c, mientras que en el segundo lo es por d y e. Lo importante es observar que la noción de pretense permite cuestionar que Oa,d,e es inconcebible. Esto será explicitado, sin discutir la plausibilidad de la esencialidad de origen, en el texto principal.
} 
tendrían condicionales de este tipo. Sin tal compromiso, Roca - Royes sostiene que se sobre generaliza (overgeneralize) la aplicación de este condicional, a casos como: "If I am sitting, I am necessarily not standing." (ROCA-ROYES, 2011, p. 32). Debiese cuestionarse, no obstante, cómo la noción de pretense permite rechazar el antecedente del condicional como verdadero, pues precisamente lo que se exige es que se pretenda que q es verdadero. No es lo que ocurre en los casos de identidad teórica, cuya información empírica permite sostener no sólo que se pretende que "Agua es $\mathrm{H} 2 \mathrm{O}$ ” es verdadero, sino que es verdadero simpliciter. Si Kripke (1970, p. 102) tuvo en consideración una tesis esencialista al sostener que

$$
\begin{aligned}
& \mathrm{P} \rightarrow \square \mathrm{P} \\
& \mathrm{P}
\end{aligned}
$$

$\square \mathrm{P}$

Es indiscutible que no es un caso de pretense (pues está fundado en información empírica, a posteriori). Más importante, es plausible sostener que:

v) La esencialidad no trivial es una consecuencia de información empírica que descarta ciertas proposiciones como expresando una posibilidad de re, en este caso $\neg \mathrm{P}(\mathrm{y} \square \neg \mathrm{P})$.

Nuevamente, se observa cómo la tesis esencialista es consecuencia, no base de lo necesario a posteriori. Considerando el análisis previo, puede afirmarse del experimento mental que:

vi) El experimento no cumple un rol evidencial a favor de la tesis kripkeana de lo necesario a posteriori. No es la base de la necesidad a posteriori, y su rol es de carácter heurístico.

En efecto, la base epistémica de un experimento mental es a priori. Si bien se apoya en la información empírica disponible, no entrega nuevos datos, y su importancia (al menos en el caso de la tierra gemela) reside en el énfasis en la distinción (y separación) del nexo concebible - posible.

\subsection{DrY EARTh}

Un experimento mental puede ser modificado. Es lo que realiza Besson (2012), siguiendo a Boghossian (1997) y Segal (2000). Debido a que tal escenario contrafáctico descansa en un supuesto similar al cuestionado líneas 
arriba, conviene considerar las consecuencias que puede tener el asumir como correcto que el experimento mental propuesto por Putnam (1975) es un fundamento de enunciados de identidad teórica ${ }^{18}$. El experimento mental de la tierra seca tiene por objetivo cuestionar cómo el externalismo puede dar razón del significado de términos de clase, si es el caso que dicha clase es vacía. Esto es ambiguo, pues una clase C, tal como acepta Besson (2012, p. 406) puede ser vacía de dos modos:

"(i) An empty natural kind term is a term that fails to apply to any sample.

(ii) An empty natural kind term is a term that fails to refer to any natural kind!”.

Besson (2012, p. 406, n. 7) sostiene que comprende los términos de clase natural como términos generales, no singulares. Por razones discutidas en otro escrito (MIRANDA, 2012) esto parece implausible. Es correcto, como observa Besson, que los términos de clase natural cumplen el rol predicativo: "[...] general terms are syntactically words that can form noun-phrases, be preceded in English by (in)definite articles, quantifiers and attributive adjectives." (BESSON, 2012, p. 406).

Pero en tales casos, el término de clase natural no cumple el rol referencial, sino el que denomina el autor de aplicarse a (applying to). Una de las razones principales por las que un término de clase debe ser comprendido en su rol referencial como singular, es debido a que de este modo es viable cumpla el rol de término rígido de jure, tesis que comparte Besson (2010). Tal vez más importante para este escrito, si no se sostiene que un término de clase natural es singular, no se comprende como afirmar (ii), pues habría una dependencia del rol referencial con el rol predicativo: "Natural kind terms have two sorts of basic semantic functions: referring to kinds and, because they are general terms, applying to, or being true of, the samples of the kinds they refer to." (BESSON, 2012, p. 406)

Besson es explícito, por tanto, en que el único modo en qué un término general (como lo es un término de clase natural según el autor) refiera, es

\footnotetext{
${ }^{18}$ Ver Besson (2012, p. 403 - 404): "Externalists and internalists disagree about whether the meanings of natural kind terms are partly individuated by what exists in the external physical environment of the speakers using these terms." El experimento mental de la tierra seca tiene por objetivo cuestionar la tesis externalista. Pero si se sostiene, como se intenta en este escrito, que el experimento mental no cumple sino un rol heurístico, no es viable establecer una crítica a la tesis externalista bajo el supuesto de que el experimento mental justifica esta tesis inicial.
} 
que tenga casos (samples) a los que se aplique. Pero, si esto es correcto, la distinción de modos de ser vacío es inviable, pues dicha distinción supone una independencia de ambos roles. Específicamente, (i) sería inviable, pues: ¿Qué término de clase T no cumpliría el rol predicativo, si es este rol el que determina que sea un término de clase? Respecto al caso (ii), cabe considerar las consecuencias del experimento de la tierra seca, particularmente ante la siguiente cuestión: ¿Requiere este experimento que la clase C sea vacía

a) en el mundo actual

O

b) sólo en un mundo posible?

Tanto en a) y b) (considerados por separado), (ii) no se cumple: la clase no es estrictamente vacía. Intuitivamente, sólo se cumple si es vacía en ambos casos. Sin embargo, esto no es lo que supone el experimento de la tierra seca, sino que asume (pues no puede ser negado a partir de la información a posteriori del mundo actual) que agua es una clase vacía en un mundo posible, no en el mundo actual. ${ }^{19}$ Análogo al experimento mental de la tierra gemela, el objetivo aquí es cuestionar si el significado del término "agua" cambia, si su extensión cambia. ${ }^{20}$ Sin embargo, esto supone que la extensión determina

\footnotetext{
${ }^{19}$ Ver Besson (2012, p. 408): "In a Dry-Earth scenario, we compare Earth, where 'water' refers to water, and Dry-Earth, where (apparently at least) 'water' refers to nothing whatsoever-where there is (apparently) no natural kind that 'water' refers to."

${ }^{20}$ Específicamente, si el témino "agua" refiere otra clase de líquido distinta, pero con similar estereotipo: "...the issue is whether 'water' would mean something different, if it referred to a different liquid with the same stereotype (on Twin-Earth) from that which it actually refers to (on Earth).” (BESSON, 2012, p. 408). En última instancia, esto deriva en el problema de la trivialización de la rigidez, como destaca Haukioja (2012, p. 401): “[...] general terms designate their extensions, but combining this assumption with Kripke's definition (Nota de quién escribe: la definición de rigidez) will clearly not do. We would then be claiming that rigid general terms have the same extension across possible worlds, and it is easy to see the problem. On this view, most general terms, including natural kind terms, would come out non-rigid. For example, there could be more or fewer cats than there actually are." El problema de la trivialización de la rigidez será desarrollado en otro escrito. Baste enfatizar aquí que es uno de los motivos por los que Besson (2010, p. 25) defiende la rigidez de jure en los términos de clase natural: "natural kind terms come out as rigid, but so do all other general terms, and in particular all descriptive general terms." La trivialización sería un argumento contra la utilidad de la noción de rigidez como criterio diferenciador de enunciados necesarios a posteriori. Junto con esta noción de trivialización se encuentra la denominada sobre generalización (overgeneralization). Usualmente comprendidas como el mismo problema, lo cierto es que la sobre generalización no tiene las mismas consecuencias epistémicas y metafísicas que las que se atribuyen a los casos de trivialización. El uso indiferenciado de estas nociones es un error: es viable admitir sobre generalización sin derivar en trivialización de la rigidez. Esto es particularmente importante, si se tiene en consideración la tesis intuitivamente correcta de que los denominados enunciados de identidad teórica, en tanto enunciados
} 
el significado de los términos de clase natural, tesis implausible, entre otras razones, por exigir que la relación uno - uno (designación) dependa de la relación uno - muchos (denotación). ${ }^{21}$

\section{A POSTERIORI Y ESENCIALISMO CIENTÍFICO}

Se ha explicitado anteriormente cómo la exigencia de a posterioridad debiese recaer en los términos de la derecha del enunciado de identidad en cuestión. Beebee y Nigel Sabbarton-Leary $(2010,162)$ aceptan como una característica de de estos términos el ser rígidos de facto ¿Qué diferencia a un término rígido de facto de un "descriptor"? Tal vez más importante: ¿Es un término rígido de facto un caso de lo que se denominan Strongly Rigid Designator? Estas preguntas son centrales, pues la crítica de los autores a estos términos es que, a fin de cuentas, no hay un criterio que impida descartar la tesis de que son términos estipulados, con la consecuencia de que, de este modo, no serían enunciados necesarios a posteriori, sino a priori. Los autores sostienen:

[...] it might be that the relevant rigid designator on the left of our theoretical 'identification' is introduced as a matter of stipulative definition, just as a bachelor is defined to be an unmarried man. Or it might be that it is what we shall call a 'descriptor': a designator that has descriptive content that uniquely identifies the kind in question. Either way, the truth in question will be necessary but knowable a priori. (BEEBEE; SABBARTON-LEARY, 2010, p. 161).

de identidad, no debiesen ser comprendidos como un caso especial de estos. En otras palabras, la sobre generalización de la rigidez admitiría casos de enunciados de identidad teórica que hasta el momento serían rechazados, al entenderlos como un caso de trivialización.

${ }^{21}$ Este punto es discutido en detalle en Miranda (por venir). El punto central es que la determinación del significado de términos de clase natural no requiere de casos (samples) en todo (ni cada uno) mundo posible: la tierra seca no es un problema para la determinación del término de clase natural "agua", si se asume que en el mundo actual si tiene casos. Un problema ulterior es si en el mundo actual no hay casos de agua. Sobre este punto, Besson (2010, p. 423) sostiene: “[...] suppose 'water' is empty on Earth [in the sense of $\left(i^{*}\right)$ or (ii)]. Do we really have the intuition that 'water' would then mean something different than it actually does? At least the intuition is not as clear cut as in the Twin-Earth case: you might think that 'water' would be meaningful, but perhaps not that its meaning would be different. If so, it seems that the intuition of difference of meaning in the standard Twin-Earth case depends crucially on the different sorts of samples that we find on Earth and Twin-Earth-they have different molecular structures." Esto es otorgarle un rol evidencial al experimento mental de la tierra gemela, pues asume que esta clase "Twater" (cuya composición sería XYZ) entrega un dato empírico, lo que es contraintuitivo. Puede observarse lo complejo que resulta sostener que los enunciados necesarios a posteriori dependan del experimento mental. 
La característica principal de ambas alternativas enunciadas es que no cumplen con la exigencia de a posterioridad. Definiciones del tipo "soltero es definido como hombre no casado" son plausiblemente comprendidas como a priori. Y de ello los autores observan que: "[...] the essence of the kind is not simply the meaning of the kind term." (BEEBEE; SABBARTON-LEARY, 2010 , p. 161). La intuición de fondo en esta afirmación es que si este fuese el caso, si la esencia fuese el significado del término de la izquierda, sería a priori. No obstante, esto es errado, pues precisamente uno de los objetivos de la extensión de la rigidez a los términos de clase natural es establecer el significado de estos términos, sin que esto derive en la tesis fuerte de que la determinación de propiedades esenciales exige estipulación a priori, ni que sea tampoco lo que establezca la necesidad de los enunciados necesarios a posteriori. Como se ha observado al inicio de este escrito, uno de los objetivos esencialistas kripkeanos es permitir que los enunciados necesarios a posteriori fundamenten este esencialismo. Conviene recordar qué entiende Kripke por un término rígido de facto: [...] de facto rigidity [...] a description the $\mathrm{x}$ such that Px' happens to use a predicate "P" that in each possible world is true of one and the same unique object (e.g., "the smallest prime" rigidly designates the number two). (KRIPKE, 1980, p. 21, n.21).

Parece correcto sostener que la principal diferencia entre un descriptor y un término rígido de facto es que el primero permite estipulaciones descriptivas de clases (u objetos) no descubiertos, de modo tal que efectivamente el criterio de a posterioridad sería vulnerado. El ejemplo de los autores es el de "ununbium", un caso paradigmático de estipulación descriptiva a priori: "ununbium is the element with atomic number 112" (BEEBEE; SABBARTON-LEARY, 2010, p. 161). Los autores proponen que casos como éste, determinados por una estipulación definida por la IUPAC (International Union of Pure and Applied Chemistry) son analíticos ¿Por qué no ocurre lo mismo con "oro es el elemento cuyo número atómico es 79"? Porque el número atómico de "ununbium” es una determinación a priori de una clase meramente estipulada. Por contraparte, no parece un asunto de mera estipulación el que el predicado "el menor primo" designe rígidamente el número 2, o más importante, que "oro es el elemento con número atómico 79 ” no requiera de cierta información empírica que justifique (y no que sólo estipule) que el enunciado es verdadero.

Por su parte, el esencialismo científico (Scientific Essentialism) defendido por Brian Ellis (2001) sostiene que las leyes naturales se comprenden como metafísicamente necesarias, y cognoscibles sólo a posteriori. Esto sería una 
consecuencia de sostener que hay necesidad de re en enunciados que incluyen términos que designan clases naturales. Coincide también con la comprensión que tiene Kripke (1980) de los enunciados de identidad teórica, al afirmar que son metafísicamente necesarios. Esto se observa ya en su primera conferencia:

[...] if something is false, it's obviously not necessarily true. If it is true, might it have been otherwise? Is it possible that, in this respect, the world should have been different from the way it is? If the answer is 'no', then this fact about the world is a necessary one. If the answer is 'yes', then this fact about the world is a contingent one. This in and of itself has nothing to do with anyone's knowledge of anything. (KRIPKE, 1980, p. 38).

La admisión de hechos necesarios es fundamental para la ulterior afirmación de que los enunciados de identidad son necesarios de re. Si bien es cierto el objetivo central de Ellis es sostener que las denominadas disposiciones corresponde a la esencia de las clases naturales en discusión, y de este modo derivar necesidad natural de la denominada necesidad metafísica ${ }^{22}$, este es un paso allende los objetivos de este escrito. Para efectos de este escrito basta considerar en qué medida el esencialismo propuesto por Ellis descansa en condiciones de carácter a priori (ELLIS, 2001, p.19-21). ${ }^{23}$ De este modo, no afecta la comprensión de lo necesario a posteriori, pero sí permite su defensa ulterior. Como se ha dicho, Brian Ellis (2001) defiende un esencialismo disposicional, esto es una consecuencia de su propuesta de clases naturales disposicionales (dynamics kinds). Aquí se rescata otro aspecto del esencialismo científico propuesto por Ellis: El nexo entre clase sustancial - clase propiedad. Independiente de la plausibilidad del disposicionalismo, la distinción clase sustancial - clase propiedad permite sostener que los enunciados de identidad teórica son la expresión de la relación entre estas dos clases:

22 Ver Beebee; Sabbarton-Leary (2010, p. 171). Los autores sostienen que para Ellis (2001, p. 248): “[...] 'natural necessities' are 'grounded in the world'.” Ver también LE GALL (2013, p. 137), quien defiende un nexo entre disposicionalismo - microestructuralismo: "[...] podemos identificar la propiedad 'disposicional' (ser venenoso) con la micro-estructura (bioquímica) correspondiente. Solo necesitamos saber que existe una fórmula química dada para traducir en qué consiste lo venenoso." Una tesis de este tipo exige un compromiso con propiedades que supervienen (supervene).

${ }^{23}$ Allí, el autor enuncia las condiciones para ser una clase natural K, a saber: (1) be objective (i.e. mind-independent); (2) be categorically distinct, having no ontologically vague boundaries ; (3) be demarcated from all other kinds via its intrinsic properties; (4) allow for species variation, where, for instance, two isotopes are members of the same element-kind in virtue of possessing the relevant essence (atomic number), while nevertheless differing from one another intrinsically by having a distinct atomic mass.; (5) form a species-to-genus hierarchy in cases where a particular is a member of two (or more) natural kinds; (6) have an (intrinsic) essence that is both necessary and sufficient for kind membership. 
Clase sustancial - término rígido de jure

Clase propiedad - término rígido de facto.

Como observa acertadamente Bealer (2002, p. 108), se requiere el criterio de unicidad entre estas dos clases para descartar identidades contingentes, o co - referencialidad simpliciter, a diferencia de coreferencialidad rígida. Esto permite sostener, como ha intentado este escrito, que las condiciones esencialistas no triviales no fundamentan los enunciados necesarios a posteriori.

\title{
5 Conclusión
}

Los enunciados de identidad teórica son un caso de enunciados de identidad. La exigencia de self identity parece ser la condición que estos enunciados deben cumplir. Del mismo modo, la co - referencialidad rígida permite sostener las condiciones semánticas que estos enunciados deben cumplir. Por otra parte, el experimento mental de la tierra gemela no exige un compromiso ontológico con el nexo concebible - posible, motivo por el que se sostiene el rol heurístico responde de mejor modo al objetivo central de dicho experimento mental. De este modo, se evita la crítica que fundamenta el abuso enunciado por Beebee y Nigel Sabbarton-Leary (2010). Finalmente, se sostuvo como el esencialismo científico, al descansar en tesis de carácter $a$ priori, no afectan la fundamentación de lo necesario a posteriori.

ROJAS, Rafael Miranda. On the abuse of the necessary a posteriori. Tans/formlação, Marília, v. 40, n. 1, p. 211-234, Jan./Mar., 2017.

\begin{abstract}
This paper discusses how the so-called "abuse of the necessary a posteriori", proposed by Beebee and Sabbarton-Leary (2010), erroneously assumes that: a) necessary a posteriori statements require a metaphysical commitment to a non-trivial essentialism, and b) the thought experiment of the twin earth fulfills an evidential role in determining the necessary a posteriori. Against a), it is argued that the necessary a posteriori does not require a non-trivial essentialist commitment; at most, nontrivial essentialism is a consequence of the necessary a posteriori. Contra b), it is stated how the thought experiment of the twin earth fulfills a subsidiary, explanatory, and non-evidential role. This prevents an ontological commitment to the conceivable-possible connection. It is then discussed how the notion of rigid coreferentiality (de jure-de facto) is a way of solving the non-trivial essentialism requirement. Finally, we consider how Brian Ellis' scientific essentialism, in stating a priori conditions of satisfaction, does not affect the necessary a posteriori justification.
\end{abstract}

Keywords: Necessity. A posteriori. A priori. Essentialism. Thought experiment. 


\section{REFERÊNCIAS}

BEALER, G. Modal epistemology and the rationalist renaissance. In: GENDLER, T. S.; HAWTHORNE, J. (Ed.). Conceivability and possibility. Oxford: Oxford University Press, 2002. p. 71-125.

BEEBEE, H.; SABBARTON-LEARY, N. On the abuse of the necessary a posteriori. In:______ (Ed.). The semantics and metaphysics of natural kinds. New York: Routledge, 2010. p. 159-179.

BOGHOSSIAN, P.; PEACOCKE, C. (Ed.). New essays on the a priori. Oxford: Clarendon, 2000.

BROWN, J.; FEHIGE, Y. Thought experiments. In: The Stanford encyclopedia of philosophy. Stanford: Stanford University, 2011. Disponível em: <https://plato.stanford. edu/entries/thought-experiment/>. Acesso em: 10 out. 2016.

CASUlLO, A. A Priori justification. Oxford: Oxford University Press, 2003.

CHANG, H. Is water H2O? Evidence, realism and pluralism. Dordrecht: Springer, 2012.

CONNELLY, N. G. et al. Nomenclature of inorganic chemistry: IUPAC recommendations 2005. Cambridge: Royal Society of Chemistry, 2005.

DELLA ROCCA, M. Essentialism versus essentialism. In: GENDLER, T. S.;

HAWTHORNE, J. (Ed.). Conceivability and possibility. New York; Oxford: Clarendon; Oxford University Press, 2002. p. 223-252.

ELLIS, B. Scientific essentialism. Cambridge: Cambridge University Press, 2001.

GENDLER, T. S.; HAWTHORNE, J. (Ed.). Conceivability and possibility. New York; Oxford: Clarendon; Oxford University Press, 2002.

GLÜER, K.; PAGIN, P. General terms and relational modality. Nô̂s, v. 46, n. 1, p.159$199,2012$.

KANT, I. Critique of pure reason. Tradução de Norman Kemp Smith. New York: St. Martin, 1965.

KRIPKE, S. Identity and necessity. In: MUNITZ, M. K. (Ed.). Identity and individuation. New York: New York University Press, 1971. p. 135-164.

KRIPKE, S. Naming and necessity. Cambridge, MA: Harvard University Press, 1980.

LE GALL, S. Términos de clases naturales y entes posibles: algunos problemas de accesibilidad referencial. Discusiones Filosóficas, v. 14, n. 23, p. 135-153, 2013.

MCLEOD, S. Rationalism and modal knowledge. Critica, v. 41, n. 122, p. 29-42, 2009.

MIRANDA, R. Rigidez, esencialismo y actualismo en los términos referenciales para clases naturales. Discusiones Filosóficas, Manizales, Colombia: Universidad de Caldas, año 12, n. 19, p. 181-198, 2011. 
. Rigidez de jure y de facto en los términos generales para clases naturales. Areté, v. XXIV, n. 1, p. 57-90, 2012.

Enunciados de identidad, invariabilidad proposicional y estipulación contextual. Discusiones Filosóficas, v. 14, n. 23, p. 105-133, 2013.

Enunciados necesarios a posteriori, necesidad débil y racionalismo. Ideas y Valores, Bogotá, Colombia, v. 65, n. 160, p. 49-74, abr. 2016.

PERCIVAL, P. Theoretical terms: meaning and reference. In: NEWTON-SMITH, W. H. (Ed.). A companion to the philosophy of science, 2001. p. 495-514.

POPPER, K. On the use and misuse of imaginary experiments, especially in quantum theory. In: __ . The logic of scientific discovery. Londres: Hutchinson, 1959. p. 442456.

PUTNAM, H. Meaning and reference. Journal of Philosophy, v. 70, n. 19, p. 699-711, 1973.

ROCA-ROYES, S Conceivability and De Re modal knowledge. Noûs, v. 45, n. 1, p.2249, 2011.

WORLEY, S. Conceivability, possibility and physicalism. Analysis, v. 63, n. 1, p. 15-23, 2003.

YABLO, S. Is conceivability a guide to possibility? Philosophy and Phenomenological Research, v. 53, n. 1, p. 1-42, 1993.

Recebido em 13/09/2016

Aceito em 21/12/2016 